\title{
A single product perishing inventory model with demand interaction
}

\author{
VSS Yadavalli* CdeW van Schoor ${ }^{\dagger} \quad$ JJ Strasheim $^{\dagger} \quad$ S Udayabaskaran ${ }^{\ddagger}$ \\ Received: 30 August 2004; Revised: 30 September 2004; Accepted: 5 October 2004
}

\begin{abstract}
The paper describes a single perishing product inventory model in which items deteriorate in two phases and then perish. An independent demand takes place at constant rates for items in both phases. A demand for an item in Phase I not satisfied may be satisfied by an item in Phase II, based on a probability measure. Demand for items in Phase II during stock-out is lost. The re-ordering policy is an adjustable $(S, s)$ policy with the lead-time following an arbitrary distribution. Identifying the underlying stochastic process as a renewal process, the probability distribution of the inventory level at any arbitrary point in time is obtained. The expressions for the mean stationary rates of lost demand, substituted demand, perished units and scrapped units are also derived. A numerical example is considered to highlight the results obtained.
\end{abstract}

Key words: Inventory modelling, perishing inventory, lead-time, re-orders, substituted demand, (S, s) policy, renewal process, product density

\section{Introduction}

In inventory models of perishing products the lifetime of the products in the inventory model is described in alternative ways. One assumption is that the product has a fixed lifetime and if no demand occurs for the product within its lifetime, it is considered perished and removed from the inventory. Nahmias (1982) has given an exhaustive survey of the fixed-life perishable inventory literature. Another description of the lifetime is that the product deteriorates continuously in quality over time and eventually perishes. Raafat (1991) has presented a review of the literature on deteriorating (decaying) inventory models. Apart from the lifetime consideration, the deteriorating items have one important kind of interaction on the demand process in the sense that, in addition to the usual demand, there may also be a separate demand for items slightly deteriorated in quality if the cost is comparatively lesser than a new one. For example, vegetables, food, meat

\footnotetext{
*Corresponding author: Department of Industrial and Systems Engineering, University of Pretoria, Pretoria, 0002, South Africa, yadavalli@postino.up.ac.za

${ }^{\dagger}$ Department of Industrial and Systems Engineering, University of Pretoria, Pretoria, 0002, South Africa

${ }^{\ddagger}$ Presidency College, University of Madras, Chennai, India
} 
and fish loose their lustre as time elapse. A day old vegetable is slightly inferior in quality compared to a fresh one. Such items may be accepted by some customers in the event of non-availability of new fresh ones. There may also be a significantly different demand for slightly deteriorated items due to the fact that they are less expensive. Two-product continuous review inventory models have been studied recently by Yadavalli et al. (2001), Yadavalli \& Joubert (2003) and Yadavalli et al. (2004).

In this paper, an attempt is made to incorporate the above kind of interaction in the study of deteriorating product inventory systems. Specifically, a continuous review of perishing inventory models is considered with the assumption that if there is no demand for a product in inventory, it passes through two phases and then perishes. An item in Phase I is fresh and in Phase II slightly deteriorated. On leaving Phase II, it is considered perished and removed from inventory or scrapped. Independent demand takes place at constant rates for items in both phases. Demand for an item during Phase I, stock-out may be satisfied by an item in Phase II based on a probability measure. Demand for product in phase II during stock-out is lost. Using the regeneration point technique, various measures of the inventory model are obtained.

The organization of this paper is as follows: $\S 2$ lists various assumptions and notations in the description of the inventory model and also provides the auxiliary functions which are needed to describe the behaviour of the process between two successive regeneration points of the underlying stochastic process describing the inventory model. Various performance measures of the inventory model are obtained in $\S 3$. A cost analysis is provided in $\S 4$ and some numerical results are presented in $\S 5$.

\section{Assumptions and auxiliary function}

We consider a continuous inventory model under the following assumptions:

1. The item under consideration is perishable.

2. The lifetime distribution of an item is a generalized Erlang distribution with two phases. For the sake of convenience, the items in Phase I are designated as Product 1 and that in Phase II as Product 2.

3. The demand for product $\mathrm{i}$ occurs independently with constant rate $\lambda_{i}, i=1,2$.

4. Maximum storage capacity or total capacity of the inventory level is $S$ and re-order takes place if the total inventory level is $s$.

5. At the epoch of replenishment, all items of Product 2 are scrapped (deleted) and the inventory level is raised to $S$.

6. The lead-time is arbitrary with probability density function $f($.$) , and survivor func-$ tion $\bar{F}(t)=1-F(t)$.

7. A demand for Product 1 occurring during the stock-out period can be substituted by an item of Product 2 with probability $p$ if available, $0 \leq p \leq 1$. 
8. A demand for Product 2 occurring during the stock-out period is lost, that is no backlogging is possible.

The following notation is used:

$a_{j}$ : Event that a re-order takes place when the inventory level of Product 2 is $j, 0 \leq j \leq s$.

$a:$ Any $a_{j}$-event, $0 \leq j \leq s$.

$r_{i j}$ : Event that a stock replenishment occurs. $S-i$ units of Product 1 are added to the inventory and $j$ units of Product 2 scrapped from the inventory.

$r:$ Any $r_{i j}$-event, $0 \leq i, j ; i+j \leq s$.

$l_{j}: \quad$ Event that a demand for product $j$ is lost, $j=1,2$

$g:$ Event that a demand for Product 1 is substituted by Product 2 .

$d_{i}$ : Event that a demand for product $i$ is satisfied with product $i, i=$ 1,2 .

$k_{1}$ : Event of Product 1 transitting as Product 2.

$k_{2}$ : Event of Product 2 perishing and being removed from the inventory.

$L_{i}(t)$ : Inventory level of product $i$ at time $t ; i=1,2$.

$Z(t): \quad\left(L_{1}(t), L_{2}(t)\right)$.

$\lambda_{i}: \quad$ The demand rate of product $i, i=1,2$.

$\mu_{i}$ : The perishing rate of product $i, i=1,2$.

$N(\eta, t):$ Number of $\eta$ events in $(0, t]$.

$E\left[N\left(a_{j}, \infty\right)\right]:$ The mean stationary rate of re-order.

$E\left[N\left(k_{1}, \infty\right)\right]$ : The mean stationary rate of transit of Product 1 as Product 2 .

$E\left[N\left(k_{2}, \infty\right)\right]$ : The mean stationary rate of perishing and removed from the inventory.

$C R$ : Re-ordering cost.

$C L_{i}: \quad$ Cost of lost demand for product $i, i=1,2$.

$C P$ : Salvage cost per scrapped (deleted) unit.

$C B$ : Purchase price of one unit.

$C(S, s)$ : Total expected cost per unit time.

(C): Convolution symbol.

$f^{*}(\theta)$ : Laplace transform of $f(t)$,

In order to study the stochastic process $\left(L_{1}(t), L_{2}(t)\right)$, we first note that the $r$-events constitute a renewal process (see Figure 1 below). Consequently, it is sufficient to describe the behaviour of the inventory process between two successive renewals. Therefore, we introduce some auxiliary functions.

\subsection{The function $P(k, l, t \mid i, j)$}

We define $P(k, l, t \mid i, j)=P[Z(t)=(k, l), N(\eta, t)=0 \mid Z(0)=(i, j)], \eta=a, r$ to represent the probability distribution of the inventory level in an interval in which neither re-order nor replenishment can occur. To derive an expression for this function, we note that a change in the inventory level may occur due to any one of the following possibilities: 


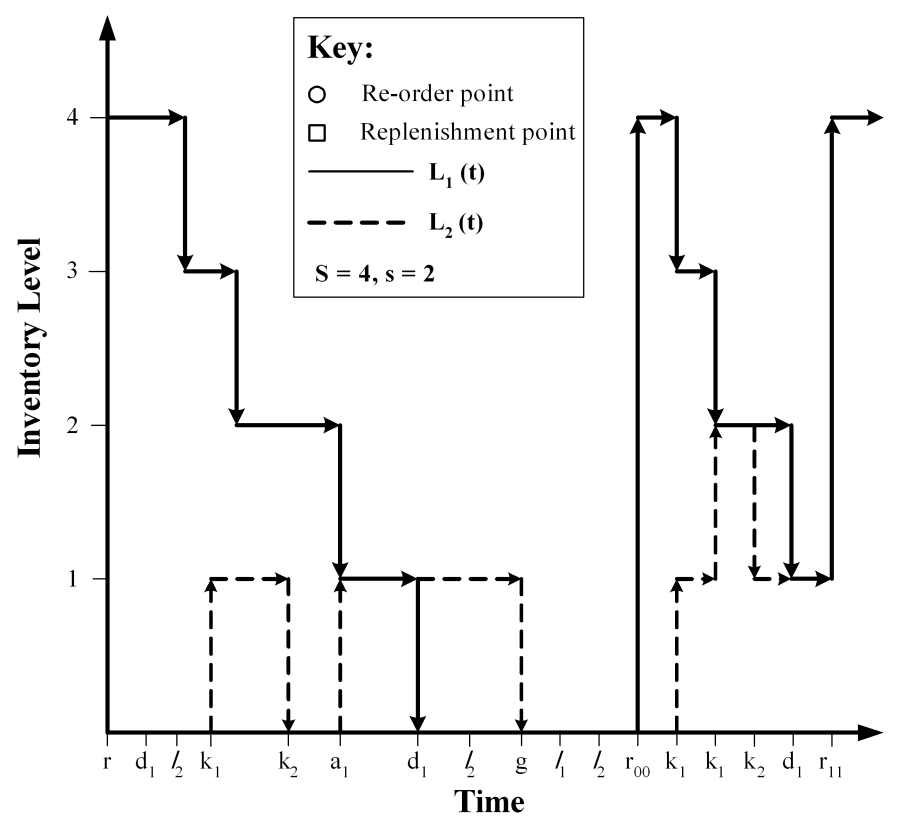

Figure 1: Realization of Events.

1. A demand for product $i$ occurs and is satisfied by product $i, i=1,2$.

2. A unit of Product 1 perishes and transits as Product 2.

3. A unit of Product 2 perishes.

4. A demand for a unit of Product 1 occurs during the stock-out period and is substituted by Product 2 with probability $p$ if it is available.

Accordingly, we have for $0 \leq k+l \leq i+j \leq s$ or $s+1 \leq k+l \leq i+j \leq S$ :

Case $1(k>i): P(k, l, t \mid i, j)=0$.

Case $2(i>0, j>0, \quad 0<k<i, \quad k+l<i+j)$ :

$$
\begin{gathered}
P(k, l, t \mid i, j)=\lambda_{1} e^{-\left(\lambda_{1}+\lambda_{2}+i \mu_{1}+j \mu_{2}\right) t}\left(\mathcal{C} P(k, l, t \mid i-1, j)+i \mu_{1} e^{-\left(\lambda_{1}+\lambda_{2}+i \mu_{1}+j \mu_{2}\right) t}\right. \\
\text { (C) } P(k, l, t \mid i-1, j+1)+\left(\lambda_{2}+j \mu_{2}\right) e^{-\left(\lambda_{1}+\lambda_{2}+i \mu_{1}+j \mu_{2}\right) t}(P(k, l, t \mid i, j-1) .
\end{gathered}
$$

Case $3(i>0, j=0,0 \leq k<i, l \geq 0, k+l<i)$ :

$$
\begin{aligned}
P[k, l, t \mid i, 0]= & \lambda_{1} e^{-\left(\lambda_{1}+\lambda_{2}+i \mu_{1}+j \mu_{2}\right) t}(P[k, l, t \mid i-1,0] \\
& +i \mu_{1} e^{-\left(\lambda_{1}+\lambda_{2}+i \mu_{1}+j \mu_{2}\right) t}(P[k, l, t \mid i+1] .
\end{aligned}
$$

Case $4(i>0, j>0, k=i, l=j): P[i, j, t \mid i, j]=e^{-\left(\lambda_{1}+\lambda_{2}+i \mu_{1}+j \mu_{2}\right) t}$.

Case $5(i>0, j>0, k=i, 0 \leq l<j)$ :

$$
P[i, l, t \mid i, j]=\left(\lambda_{2}+j \mu_{2}\right) e^{-\left(\lambda_{1}+\lambda_{2}+i \mu_{1}+j \mu_{2}\right) t}(\mathcal{C} P[i, l, t \mid i, j-1] .
$$

Case $6(i>0, j=0, k=i, l=0): P[i, 0, t \mid i, 0]=e^{-\left(\lambda_{1}+i \mu_{1}\right) t}$. 
Case $7(i=0, j>0, k=0, l=j): P[0, j, t \mid 0, j]=e^{-\left(\lambda_{1}+\lambda_{2}+j \mu_{2}\right) t}$.

Case $8(i=0, l \geq 0, k=0, l<j)$ :

$$
P[0, l, t \mid 0, j]=\left(\lambda_{1} p+\lambda_{2}+j \mu_{2}\right) e^{-\left(\lambda_{1} p+\lambda_{2}+j \mu_{2}\right) t}(P[0, l, t \mid 0, j-1] .
$$

Case $9(i=j=k=l=0): P[0,0, t \mid 0,0]=1$.

\subsection{The function $\phi_{j}(t)$}

We define

$$
\phi_{j}(t)=\lim _{\Delta \rightarrow 0} \frac{P\left[a_{j}-\text { event during }(t, t+\Delta), N(r, t)=0 \mid r-\text { event at } t=0\right]}{\Delta} .
$$

The function $\phi_{j}(t) d t$ represents the probability that an $a_{j}$-event occurs during the interval $(t, t+\Delta)$ and there is no replenishment during the interval $(0, t]$, given that an $r$-event has occurred at $t=0$. Hence, we have $\phi_{j}(t)=P[k+1, j, t \mid S, 0] \lambda_{1} \bar{F}(t)+P[k, j+$ $1, t \mid S, 0]\left[\lambda_{2}+(j+1) \mu_{2}+\delta_{k 0} \lambda_{1} p\right] \bar{F}(t)$, where $k+j=s, 0 \leq k, j \leq s$.

\subsection{The function $W(i, j, t)$}

We define $W(i, j, t)=P[Z(t)=(i, j), N(r, t)=0 \mid Z(0)=(S, 0)]$. Then the function $W(i, j, t)$ represents the probability that the inventory level is $(i, j)$ at the time $t$, where $t$ is the time elapsed since the last renewal. To obtain $W(i, j, t)$, we consider two mutually exclusive cases.

Case $1(0 \leq i+j \leq s)$ : In this case, exactly one re-order is made in $(0, t)$ and it does not materialize up to time $t$. Precisely, we have

1. The system is in state $(S, 0)$ at $t=0$.

2. The system enters the state $(k, l)$ during the interval $(u, u+d u)$ where $k+l=s$ and $0<u<t$.

3. A re-order is placed during the interval $(u, u+d u)$.

4. The re-order does not materialize up to time $t$.

5. The system enters the state $(i, j)$ at time $t$.

Using probabilistic arguments, we have

$$
W(i, j, t)=\sum_{l=0}^{s} \phi_{l}(t)(\{\bar{F}(t) P(i, j, t \mid k, l)\},
$$

where $0 \leq k, l \leq s$ and $k+l=s$.

Case $2(s+1 \leq i+j \leq S)$ : In this case no re-order takes place during the interval $(0, t)$. Hence, we have $W(i, j, t)=P[i, j, t \mid S, 0]$. The steady-state probabilities of the system are given by $W(i, j)=\lim _{t \rightarrow \infty} W(i, j, t)$. 


\section{Measures of system performance}

To obtain explicit expressions for various performance measures of the model presented in $\S 2$, we proceed to define the first-order product density

$$
h_{\eta}(t)=\lim _{\Delta \rightarrow 0} P[\eta-\text { event during }(t, t+\Delta) \mid Z(0)=(S, 0)] / \Delta,
$$

where $\eta=r, r_{i j}, a, a_{j}, d_{1}, d_{2}, l_{1}, l_{2}, g, k_{1}, k_{2}$.

\subsection{Mean number of re-orders}

Since a re-order is defined as an $a_{j}$-event, we derive expressions for $h_{i j}(t)$ to obtain the mean number of re-orders. We note that a re-order takes place when the total inventory level enters $s$. Hence, we have

$$
h_{a_{j}}(t)=\sum_{i+j=s}^{\infty}\left[W(i+1, j, t) \lambda_{1}+W(i, j+1, t)\left\{\delta_{i 0} \lambda_{1} p+\lambda_{2}+(j+1) \mu_{2}\right\}\right] .
$$

The mean number of re-orders during the interval $(0, t]$ is given by

$$
E\left[N\left(a_{j}, t\right)\right]=\int_{0}^{t} h_{a_{j}}(u) d u .
$$

Consequently, the mean stationary rate of re-orders is given by

$$
\begin{aligned}
E\left[N\left(a_{j}, \infty\right)\right] & =\lim _{t \rightarrow \infty} \frac{1}{t} E\left[N\left(a_{j}, t\right)\right]=\lim _{t \rightarrow \infty} h_{a_{j}}(t) \\
& =\sum_{i+j=s}^{\infty}\left[W(i+1, j) \lambda_{1}+W(i, j+1)\left\{\delta_{i 0} \lambda_{1} p+\lambda_{2}+(j+1) \mu_{2}\right\}\right] .
\end{aligned}
$$

\subsection{Mean number of demands for a particular product which is satisfied by the same product}

A demand for Product 1 being satisfied by Product 1 is represented by a $d_{1}$-event. Hence we derive an expression for $h_{d_{1}}(t)$. We observe that a $d_{1}$-event occurs whenever a demand for Product 1 occurs when the inventory level is $(i, j)$ where, $1 \leq i \leq S, 0 \leq j \leq S$ and $0<i+j \leq S$. Hence, we have

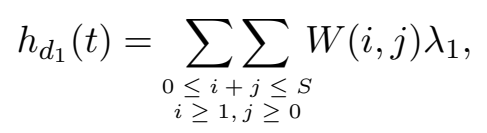

so that

$$
E\left[N\left(d_{1}, t\right)\right]=\int_{0}^{t} h_{d_{1}}(u) d u
$$

Therefore,

$$
E\left[N\left(d_{1}, \infty\right)\right]=\sum_{\substack{0 \leq i+j \leq S \\ i \geq 1, j \geq 0}} \sum_{i, j} W(i, j) \lambda_{1}
$$


In the same way, we obtain

$$
\left.h_{d_{2}}(t)=\sum_{\substack{0 \leq i+j \leq S \\ i \geq 1, j \geq 0}} \sum_{\substack{0 \\ i}} W, j, t\right) \lambda_{2}
$$

so that

$$
E\left[N\left(d_{2}, t\right)\right]=\int_{0}^{t} h_{d_{2}}(u) d u \text { and } E\left[N\left(d_{2}, \infty\right)\right]=\sum_{\substack{0 \leq i+j \leq S \\ i \geq 1, j \geq 0}} \sum_{i \geq 1} W(i, j) \lambda_{2} .
$$

\subsection{Mean number of lost demand}

A demand for Product 1 is lost when the total inventory level is zero or when the inventory level of Product 1 is zero and that of Product 2 is positive, but when the demand is not substituted with Product 2. Therefore, we have

$$
h_{l_{1}}(t)=W(0,0, t) \lambda_{1}+\sum_{j=1}^{S} W(0, j, t) \lambda_{1}(1-p)=\sum_{j=0}^{S} W(0, j, t)\left\{1-p+p \delta_{j 0}\right\} \lambda_{1} .
$$

The mean number of lost demands for Product 1 is given by

$$
E\left[N\left(l_{1}, t\right)\right]=\int_{0}^{t} h_{l_{1}}(u) d u
$$

so that the mean stationary rate of lost demand for Product 1 is given by

$$
E\left[N\left(l_{1}, \infty\right)\right]=\sum_{j=0}^{S} W(0, j)\left[1-p+p \delta_{j 0}\right] \lambda_{1} .
$$

In the same way, we have for the events $l_{2}$,

$$
h_{l_{2}}(t)=\sum_{i=0}^{S} W(i, 0, t) \lambda_{2}, \quad E\left[N\left(l_{2}, t\right)\right]=\int_{0}^{t} h_{l_{2}}(u) d u \text { and } E\left[N\left(l_{2}, \infty\right)\right]=\sum_{i=0}^{S} W(i, 0) \lambda_{2} .
$$

\subsection{Mean number of demands for product 1 substituted by product 2}

A demand for Product 1 being substituted by Product 2 is denoted by a $g$-event. We note that a $g$-event occurs during the interval $(t, t+\Delta)$ if the inventory level of the system at time $t$ equals $(0, j)$ for some $1 \leq j \leq S$, and if a demand for Product 1 occurs during the interval $(t, t+\Delta)$ being substituted by Product 2 . Hence, we have

$$
h_{g}(t)=\sum_{j=1}^{S} W(0, j, t) \lambda_{1} p \text { and } E[N(g, t)]=\int_{0}^{t} h_{g}(u) d u .
$$

Therefore,

$$
E[N(g, \infty)]=\sum_{j=1}^{S} W(0, j) \lambda_{1} p
$$




\subsection{Mean number of units deteriorated from product 1 and transitted as product 2}

Since a $k_{1}$-event pertains to the event that a unit of Product 1 deteriorates and transits as Product 2 and a $k_{1}$-event occurs during the interval $(t, t+\Delta)$ if the system is in state $(i, j)$ at time $t$ for some $1 \leq i \leq S, 0 \leq j \leq S$ and $1 \leq i+j \leq S$ and a unit in Product 1 transits as Product 2 during the interval $(t, t+\Delta)$, we have

$$
h_{k_{1}}(t)=\sum_{\substack{0 \leq i+j \leq S \\ i \geq 1, j \geq 0}} W(i, j, t) i \mu_{1}
$$

The mean number of units of Product 1 that have transitted as Product 2 during the interval $(0, t]$ is given by

$$
E\left[N\left(k_{1}, t\right)\right]=\int_{0}^{t} h_{k_{1}}(u) d u
$$

and the mean stationary rate of units of Product 1 transiting as Product 2 is given by

$$
E\left[N\left(k_{1}, \infty\right)\right]=\sum_{\substack{0 \leq i+j \leq S \\ i \geq 1, j \geq 0}} \sum_{i, j} W\left(i \mu_{1} .\right.
$$

\subsection{Mean number of units of product 2 perished and removed from the inventory}

The first order product density of $k_{2}$ is given by

$$
h_{k_{2}}(t)=\sum_{\substack{0 \leq i+j \leq S \\ i \geq 1, j \geq 0}} W(i, j, t) j \mu_{2} .
$$

Hence the mean number of units of Product 2 that have perished and are removed from the inventory during the interval $(0, t]$ is given by

$$
E\left[N\left(k_{2}, t\right)\right]=\int_{0}^{t} h_{k_{2}}(u) d u .
$$

Consequently, the mean stationary rate of perishing of units of Product 2 is given by

$$
E\left[N\left(k_{2}, \infty\right)\right]=\sum_{\substack{0 \leq i+j \leq S \\ i \geq 1, j \geq 0}} \sum_{i} W(i, j) j \mu_{2} .
$$

\subsection{Mean number of replenishments}

We consider the renewal process of $r$-events and derive its first-order product density $h_{r}(t)$. We first derive an expression for the probability density function $g(t)$ of the interval between two successive occurrences of the $r$-events. By definition, we have

$$
g(t)=\lim _{\Delta \rightarrow 0} \frac{P[r-\text { event during }(t, t+\Delta), N(r, t)=0 \mid Z(0)=(S, 0)]}{\Delta} .
$$


In order to derive $g(t)$, we easily derive its survival function $\bar{G}(t)$. Since $\bar{G}(t)$ denotes the probability that a replenishment has not occurred up to time $t$, we have two mutually exclusive cases for $\bar{G}(t)$ :

1. A re-order does not occur up to time $t$.

2. A re-order is placed during the interval $(u, u+\Delta), 0<u<t$, but it has not been realized up to time $t$.

Hence,

$$
\bar{G}(t)=\sum_{\substack{0 \leq i+j \leq S \\ i \geq 1, j \geq 0}} \sum_{\substack{1 \\ i}} P(k, l, t \mid S, 0)+\sum_{l=0}^{s} \phi_{l}(t) \Subset\left\{\bar{F}(t)+\sum_{k_{1}=0}^{s-l} \sum_{l_{1}=0}^{l} P\left(k_{1}, l_{1}, t \mid s-l, l\right)\right\} .
$$

However,

$$
h_{r}(t)=\sum_{n=1}^{\infty} g^{(n)}(t) \text { and } E[N(r, t)]=\int_{0}^{t} h_{r}(u) d u .
$$

Hence, by renewal theory, the mean stationary rate of replenishment is given by

$$
E[N(r, \infty)]=\lim _{t \rightarrow \infty} \frac{1}{t} \int_{0}^{t} h_{r}(u) d u=\frac{1}{\int_{0}^{\infty} \bar{G}(t) d t} .
$$

\subsection{Mean number of units replenished}

First, we define the product density

$$
h_{r_{i j}}(t)=\lim _{\Delta \rightarrow 0} \frac{P\left[r_{i j}-\text { event during }(t, t+\Delta) \mid Z(0)=(S, 0)\right]}{\Delta} .
$$

Next we obtain a relation between $h_{r_{i j}}(t)$ and $h_{r}(t)$. We define

$$
f_{i j}(t)=\lim _{\Delta \rightarrow 0} \frac{P\left[r_{i j}-\text { event during }(t, t+\Delta), N(r, t)=0 \mid Z(0)=(S, 0)\right]}{\Delta}
$$

and observe that

$$
\begin{aligned}
f_{i j}(t)= & \sum_{\substack{0 \leq i+j \leq S \\
i \geq 1, j \geq 0}} \sum_{\substack{1 \\
i}} P(k+1, l, t \mid S, 0) \lambda_{1}+P(k, l+1, t \mid S, 0) \\
& \left.\left\{\lambda_{2}+(l+1) \mu_{2}+\delta_{k 0} \lambda_{1} p\right\}\right] \text { (C) } f(t) P(i, j, t \mid k, l) .
\end{aligned}
$$

Consequently, we have $h_{r_{i j}}(t)=f_{i j}(t)+h_{r}(t) \Subset f_{i j}(t)$ and

$$
E\left[N\left(r_{i j}, t\right)\right]=\int_{0}^{t} h_{r_{i j}}(u) d u .
$$


Hence,

$$
E\left[N\left(r_{i j}, \infty\right)\right]=\lim _{t \rightarrow \infty} \frac{1}{t} \int_{0}^{t} h_{r_{i j}}(u) d u=E[N(r, \infty)] \lim _{\theta \rightarrow 0} f_{i j}^{*}(\theta) .
$$

Since at the occurrence of each $r_{i j}$-event, $S-i$ units of Product 1 are added to the inventory, the mean number of Product 1 items added to the inventory per unit time is given by

$$
\sum_{\substack{0 \leq i+j \leq S \\ i \geq 1, j \geq 0}} E\left[N\left(r_{i j}, \infty\right)\right](S-i)=E[N(r, \infty)] \sum_{\substack{0 \leq i+j \leq S \\ i \geq 1, j \geq 0}} \sum_{\substack{\theta \rightarrow 0 \\ i \geq 1, j}} f_{i j} *(\theta) .
$$

\subsection{Mean number of units scrapped from the inventory}

Since, at the occurrence of an $r_{i j}$-event, $j$ units of Product 2 are scrapped from the inventory per unit time, we have

$$
\sum_{\substack{0 \leq i+j \leq S \\ i \geq 1, j \geq 0}} E\left[N\left(r_{i j}, \infty\right)\right] j=E[N(r, \infty)] \sum_{\substack{0 \leq i+j \leq S \\ i \geq 1, j \geq 0}} \sum_{\substack{\theta \rightarrow 0 \\ i \geq 1}} f_{i j}^{*}(\theta) .
$$

\section{Cost analysis}

Since $E\left[N\left(l_{1}, \infty\right)\right]$ and $E\left[N\left(l_{2}, \infty\right)\right]$ are respectively the mean stationary rates of the two types of lost demands, the cost due to lost demand is given by $E\left[N\left(l_{1}, \infty\right)\right] C L_{1}+$ $E\left[N\left(l_{2}, \infty\right)\right] C L_{2}$. The cost corresponding to items of Product 2 perished and removed from the inventory is $E\left[N\left(k_{2}, \infty\right)\right] C P$. The number of items of Product 2 that are scrapped from the inventory per unit time is

$$
\sum_{\substack{0 \leq i+j \leq S \\ i \geq 1, j \geq 0}} E\left[N\left(r_{i j}, \infty\right)\right] j
$$

The cost due to this is therefore

$$
\sum_{\substack{0 \leq i+j \leq S \\ i \geq 1, j \geq 0}} E\left[N\left(r_{i j}, \infty\right)\right] j C P
$$

Hence the total expected cost per unit time is:

$$
\begin{aligned}
& C(S, s)=E[N(a, \infty)] C R+E\left[N\left(l_{1}, \infty\right)\right] C L_{1}+E\left[N\left(l_{2}, \infty\right)\right] C L_{2}+\left[E\left[N\left(k_{2}, \infty\right)\right]\right. \\
& \left.+\sum_{\substack{0 \leq i+j \leq S \\
i \geq 1, j \geq 0}} E\left[N\left(r_{i j}, \infty\right)\right] j\right] C P+\sum_{\substack{0 \leq i+j \leq S \\
i \geq 1, j \geq 0}} \sum_{i=1} E\left[\left(r_{i j}, \infty\right)\right](s-i) C B .
\end{aligned}
$$




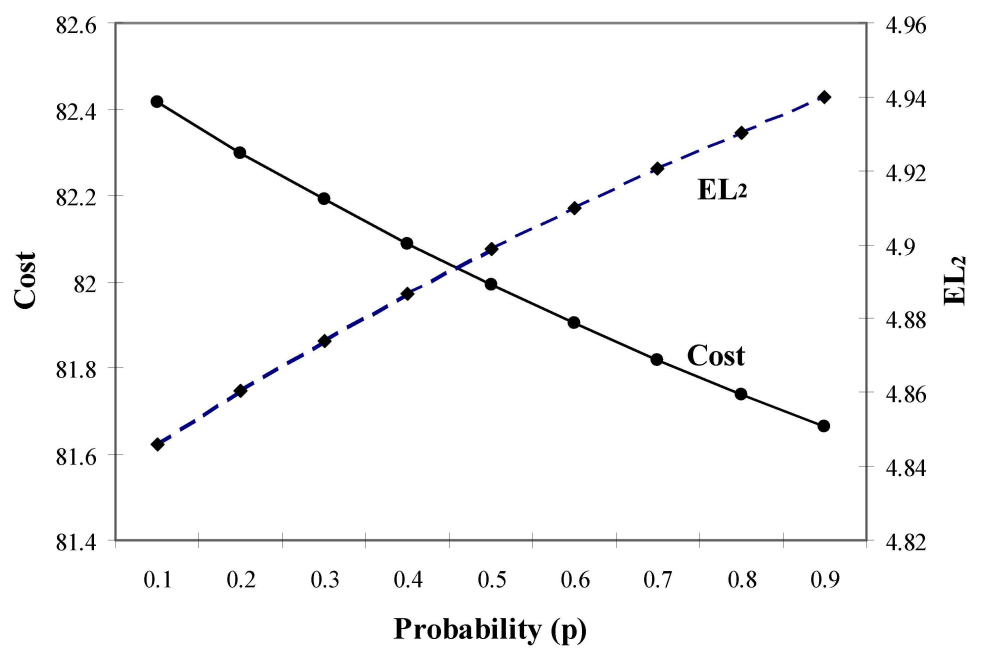

Figure 2: Relationship of COST and $E L_{2}$ versus $p$ for $S=3, s=1$.

\section{A numerical example}

For the purpose of illustration, we consider a numerical example. Let $f(t)=\theta e^{-\theta t}, t>0$, $\theta>0, \lambda_{1}=4.0, \lambda_{2}=6.0, \mu_{1}=2.5, \mu_{2}=2.5, \theta=2.0, C R=10.0, C L_{1}=6.0, C L_{2}=5.0$, $C P=4.0$, and $C B=10.0$. By varying the probability $p$ from 0.1 to 0.9 and varying $S$ from 2 to 4 , with corresponding possible values for $s$, we obtain the values, of the mean stationary rates of the following variables: (i) Demand satisfied $\left(E D_{1}, E D_{2}\right)$, (ii) Demands substituted $(E G)$, (iii) Lost demands $\left(E L_{1}, E L_{2}\right)$, (iv) Items perished $\left(E K_{2}\right)$, (v) Re-orders $(E S)$, (vi) Replenishments (RRATE), (vii) Units replenished (EUR), (viii) Units scrapped or deleted (EUS) and (ix) Total expected cost (COST).

The numerical results of the relationship between $\mathrm{p}$ and the above variables are summarized in Table 1. Per illustration, the relationships of Total Expected Cost (COST) and

\begin{tabular}{lcccccc}
\hline & $S=2, s=1$ & $S=3, s=1$ & $S=3, s=2$ & $S=4, s=1$ & $S=4, s=2$ & $S=4, s=3$ \\
\hline$E D_{1}$ & increases & increases & increases & increases & increases & increases \\
$E D_{2}$ & decreases & decreases & decreases & decreases & decreases & decreases \\
$E G$ & increases & increases & increases & increases & increases & increases \\
$E L_{1}$ & decreases & decreases & decreases & decreases & decreases & decreases \\
$E L_{2}$ & increases & increases & increases & increases & increases & increases \\
$E K_{2}$ & decreases & decreases & decreases & decreases & decreases & decreases \\
$E A$ & increases & increases & increases & increases & increases & increases \\
$R R A T E$ & increases & increases & increases & increases & increases & increases \\
$E U R$ & increases & increases & increases & increases & increases & increases \\
$E U S$ & decreases & decreases & decreases & decreases & decreases & decreases \\
$C O S T$ & decreases & decreases & decreases & decreases & decreases & decreases \\
\hline
\end{tabular}

Table 1: Relationship between $p$ and selected variables for varying $S$ and $s$.

Lost Demand $\left(E L_{2}\right)$ versus increasing values of $p$ are shown graphically in Figure 2. Detail results of the numerical example are given in Tables $2-7$ for varying values of $S$ and $s$. 


\begin{tabular}{lllllll}
\hline$p$ & $E D_{1}$ & $E D_{2}$ & $E G$ & $E L_{1}$ & $E L_{2}$ & $E K_{2}$ \\
\hline 0.1 & 1.447408 & 0.802104 & 0.030899 & 2.521693 & 5.197896 & 0.365150 \\
0.2 & 1.448016 & 0.786138 & 0.059651 & 2.492333 & 5.213861 & 0.357462 \\
0.3 & 1.448584 & 0.771246 & 0.086471 & 2.464945 & 5.228754 & 0.350288 \\
0.4 & 1.449116 & 0.757323 & 0.111549 & 2.439334 & 5.242677 & 0.343578 \\
0.5 & 1.449616 & 0.744276 & 0.135049 & 2.415335 & 5.255723 & 0.337289 \\
0.6 & 1.450087 & 0.732027 & 0.157114 & 2.392799 & 5.267973 & 0.331382 \\
0.7 & 1.450530 & 0.720503 & 0.177874 & 2.371595 & 5.279497 & 0.325824 \\
0.8 & 1.450949 & 0.709642 & 0.197440 & 2.351611 & 5.290359 & 0.320585 \\
0.9 & 1.451345 & 0.699389 & 0.215912 & 2.332742 & 5.300611 & 0.315637 \\
\hline
\end{tabular}

\begin{tabular}{llllll}
\hline$p$ & $E A$ & $R R A T E$ & $E U R$ & $E U S$ & $C O S T$ \\
\hline 0.1 & 1.523762 & 1.523762 & 2.775306 & 0.129744 & 86.0899 \\
0.2 & 1.524402 & 1.524402 & 2.776470 & 0.125240 & 85.9627 \\
0.3 & 1.525000 & 1.525000 & 2.777560 & 0.120971 & 85.8441 \\
0.4 & 1.525560 & 1.525560 & 2.778581 & 0.117015 & 85.7332 \\
0.5 & 1.526087 & 1.526087 & 2.779540 & 0.113309 & 85.6293 \\
0.6 & 1.526582 & 1.526582 & 2.780442 & 0.109832 & 85.5318 \\
0.7 & 1.527049 & 1.527049 & 2.781292 & 0.106561 & 85.4400 \\
0.8 & 1.527490 & 1.527490 & 2.782095 & 0.103480 & 85.3536 \\
0.9 & 1.527907 & 1.527907 & 2.782854 & 0.100571 & 85.2720 \\
\hline
\end{tabular}

Table 2: Numerical results for $S=2, s=1$.

\begin{tabular}{lllllll}
\hline$p$ & $E D_{1}$ & $E D_{2}$ & $E G$ & $E L_{1}$ & $E L_{2}$ & $E K_{2}$ \\
\hline 0.1 & 2.015121 & 1.154118 & 0.029509 & 1.955370 & 4.845882 & 0.591228 \\
0.2 & 2.016546 & 1.139571 & 0.057012 & 1.926442 & 4.860429 & 0.583235 \\
0.3 & 2.017882 & 1.125985 & 0.082706 & 1.899412 & 4.874015 & 0.575761 \\
0.4 & 2.019136 & 1.113267 & 0.106766 & 1.874098 & 4.886733 & 0.568758 \\
0.5 & 2.020316 & 1.101338 & 0.129342 & 1.850342 & 4.898662 & 0.562181 \\
0.6 & 2.021429 & 1.090125 & 0.150568 & 1.828003 & 4.909874 & 0.555993 \\
0.7 & 2.022480 & 1.079566 & 0.170562 & 1.806958 & 4.920434 & 0.550161 \\
0.8 & 2.023474 & 1.069605 & 0.189429 & 1.787098 & 4.930395 & 0.544654 \\
0.9 & 2.024415 & 1.060193 & 0.207260 & 1.768325 & 4.939806 & 0.539447 \\
\hline
\end{tabular}

\begin{tabular}{llllll}
\hline$p$ & $E A$ & $R R A T E$ & $E U R$ & $E U S$ & $C O S T$ \\
\hline 0.1 & 1.139148 & 1.139148 & 3.227835 & 0.104645 & 82.4150 \\
0.2 & 1.139954 & 1.139954 & 3.230117 & 0.101013 & 82.2985 \\
0.3 & 1.140709 & 1.140709 & 3.232256 & 0.097624 & 82.1897 \\
0.4 & 1.141418 & 1.141418 & 3.234266 & 0.094455 & 82.0879 \\
0.5 & 1.142085 & 1.142085 & 3.236156 & 0.091486 & 81.9924 \\
0.6 & 1.142714 & 1.142714 & 3.237938 & 0.088698 & 81.9027 \\
0.7 & 1.143308 & 1.143308 & 3.239621 & 0.086075 & 81.8182 \\
0.8 & 1.143870 & 1.143870 & 3.241213 & 0.083603 & 81.7384 \\
0.9 & 1.144402 & 1.144402 & 3.242721 & 0.081269 & 81.6631 \\
\hline
\end{tabular}

Table 3: Numerical results for $S=3, s=1$. 


\begin{tabular}{lllllll}
\hline$p$ & $E D_{1}$ & $E D_{2}$ & $E G$ & $E L_{1}$ & $E L_{2}$ & $E K_{2}$ \\
\hline 0.1 & 2.100194 & 1.209167 & 0.028260 & 1.871547 & 4.790833 & 0.619952 \\
0.2 & 2.100425 & 1.195623 & 0.054702 & 1.844873 & 4.804378 & 0.612563 \\
0.3 & 2.100644 & 1.182931 & 0.079498 & 1.819857 & 4.817068 & 0.605627 \\
0.4 & 2.100852 & 1.171014 & 0.102799 & 1.796349 & 4.828986 & 0.599103 \\
0.5 & 2.101049 & 1.159802 & 0.124737 & 1.774214 & 4.840198 & 0.592954 \\
0.6 & 2.101236 & 1.149234 & 0.145429 & 1753335 & 4.850765 & 0.587149 \\
0.7 & 2.101414 & 1.139256 & 0.164979 & 1.733607 & 4.860744 & 0.581660 \\
0.8 & 2.101583 & 1.129818 & 0.183481 & 1.714936 & 4.870183 & 0.576461 \\
0.9 & 2.101745 & 1.120878 & 0.201016 & 1.697240 & 4.879122 & 0.571530 \\
\hline
\end{tabular}

\begin{tabular}{llllll}
\hline$p$ & $E A$ & $R R A T E$ & $E U R$ & $E U S$ & $C O S T$ \\
\hline 0.1 & 2.058707 & 1.436794 & 3.566840 & 0.231180 & 94.8435 \\
0.2 & 2.058934 & 1.436952 & 3.567234 & 0.225902 & 94.7067 \\
0.3 & 2.059149 & 1.437102 & 0.567606 & 0.220951 & 94.5783 \\
0.4 & 2.059352 & 1.437244 & 3.567958 & 0.216298 & 94.4577 \\
0.5 & 2.059545 & 1.437379 & 3.568292 & 0.211917 & 94.3441 \\
0.6 & 2.059728 & 1.437507 & 3.568610 & 0.207783 & 94.2370 \\
0.7 & 2.059903 & 1.437628 & 3.568912 & 0.203878 & 94.1357 \\
0.8 & 2.060069 & 1.437744 & 3.569200 & 0.200181 & 94.0398 \\
0.9 & 2.060227 & 1.437855 & 3.569474 & 0.196677 & 93.9489 \\
\hline
\end{tabular}

Table 4: Numerical results for $S=3, s=2$.

\begin{tabular}{lllllll}
\hline$p$ & $E D_{1}$ & $E D_{2}$ & $E G$ & $E L_{1}$ & $E L_{2}$ & $E K_{2}$ \\
\hline 0.1 & 2.461397 & 1.550978 & 0.022888 & 1.515716 & 4.449023 & 0.824795 \\
0.2 & 2.462760 & 1.540001 & 0.044223 & 1.493017 & 4.460000 & 0.818603 \\
0.3 & 2.464039 & 1.529750 & 0.064159 & 1.471803 & 4.470250 & 0.812810 \\
0.4 & 2.465240 & 1.520156 & 0.082829 & 1.451930 & 4.479844 & 0.807379 \\
0.5 & 2.466372 & 1.511157 & 0.100352 & 1.433276 & 4.488843 & 0.802276 \\
0.6 & 2.467440 & 1.502699 & 0.116830 & 1.415730 & 4.497301 & 0.797472 \\
0.7 & 2.468449 & 1.494735 & 0.132354 & 1.399197 & 4.505266 & 0.792943 \\
0.8 & 2.469404 & 1.487222 & 0.147005 & 1.383592 & 4.512778 & 0.788664 \\
0.9 & 2.470309 & 1.480124 & 0.160855 & 1.368836 & 4.519876 & 0.784615 \\
\hline
\end{tabular}

\begin{tabular}{llllll}
\hline$p$ & $E A$ & $R R A T E$ & $E U R$ & $E U S$ & $C O S T$ \\
\hline 0.1 & 0.884151 & 0.884151 & 3.387431 & 0.080117 & 77.6749 \\
0.2 & 0.884640 & 0.884640 & 3.389307 & 0.077324 & 77.5813 \\
0.3 & 0.885100 & 0.885100 & 3.391067 & 0.074719 & 77.4939 \\
0.4 & 0.885531 & 0.885531 & 3.392720 & 0.072284 & 77.4120 \\
0.5 & 0.885938 & 0.885938 & 3.394278 & 0.070030 & 77.3352 \\
0.6 & 0.886321 & 0.886321 & 3.395747 & 0.067862 & 77.2629 \\
0.7 & 0.886684 & 0.886684 & 3.397136 & 0.065848 & 77.1949 \\
0.8 & 0.887027 & 0.887027 & 3.398450 & 0.063950 & 77.1037 \\
0.9 & 0.887352 & 0.887352 & 3.399695 & 0.062158 & 77.0700 \\
\hline
\end{tabular}

Table 5: Numerical results for $S=4, s=1$. 


\begin{tabular}{lllllll}
\hline$p$ & $E D_{1}$ & $E D_{2}$ & $E G$ & $E L_{1}$ & $E L_{2}$ & $E K_{2}$ \\
\hline 0.1 & 2.571096 & 1.635669 & 0.021343 & 1.407561 & 4.364332 & 0.872010 \\
0.2 & 2.571408 & 1.625571 & 0.041319 & 1.387274 & 4.374429 & 0.866307 \\
0.3 & 2.571703 & 1.616109 & 0.060056 & 1.368241 & 4.383891 & 0.860949 \\
0.4 & 2.571984 & 1.607225 & 0.077667 & 1.350350 & 4.392776 & 0.855907 \\
0.5 & 2.572251 & 1.598865 & 0.094251 & 1.333499 & 4.401135 & 0.851153 \\
0.6 & 2.572504 & 1.590985 & 0.109898 & 1.317598 & 4.409014 & 0.846663 \\
0.7 & 2.572746 & 1.583545 & 0.124684 & 1.302570 & 4.416455 & 0.842414 \\
0.8 & 2.572976 & 1.576508 & 0.138680 & 1.288344 & 4.423493 & 0.838389 \\
0.9 & 2.573195 & 1.569841 & 0.151948 & 1.274857 & 4.430159 & 0.834568 \\
\hline
\end{tabular}

\begin{tabular}{llllll}
\hline$p$ & $E A$ & $R R A T E$ & $E U R$ & $E U S$ & $C O S T$ \\
\hline 0.1 & 1.557317 & 1.082949 & 3.764555 & 0.170350 & 87.6552 \\
0.2 & 1.557506 & 1.083081 & 3.765011 & 0.166502 & 87.5522 \\
0.3 & 1.557685 & 1.083205 & 3.765444 & 0.162895 & 87.4556 \\
0.4 & 1.557855 & 1.083323 & 3.765855 & 0.159505 & 87.3647 \\
0.5 & 1.558017 & 1.083436 & 3.766245 & 0.156314 & 87.2792 \\
0.6 & 1.558170 & 1.083542 & 3.766616 & 0.155504 & 87.1984 \\
0.7 & 1.558316 & 1.083644 & 3.766970 & 0.150461 & 87.1221 \\
0.8 & 1.558456 & 1.083741 & 3.767308 & 0.147770 & 87.0498 \\
0.9 & 1.558589 & .083834 & 3.767629 & 0.145219 & 86.9813 \\
\hline
\end{tabular}

Table 6: Numerical results for $S=4, s=2$.

\begin{tabular}{lllllll}
\hline$p$ & $E D_{1}$ & $E D_{2}$ & $E G$ & $E L_{1}$ & $E L_{2}$ & $E K_{2}$ \\
\hline 0.1 & 2.639271 & 1.675820 & 0.020484 & 1.340245 & 4.324180 & 0.902930 \\
0.2 & 2.639348 & 1.666208 & 0.039680 & 1.320971 & 4.333792 & 0.897530 \\
0.3 & 2.639421 & 1.657192 & 0.057710 & 1.302868 & 4.342807 & 0.892449 \\
0.4 & 2.639492 & 1.648719 & 0.074677 & 1.285831 & 4.351282 & 0.887662 \\
0.5 & 2.639560 & 1.640739 & 0.090675 & 1.269766 & 4.359261 & 0.883141 \\
0.6 & 2.639623 & 1.633211 & 0.105786 & 1.254591 & 4.366789 & 0.878865 \\
0.7 & 2.639685 & 1.626096 & 0.120081 & 1.240234 & 4.373904 & 0.874815 \\
0.8 & 2.639744 & 1.619362 & 0.133628 & 1.226628 & 4.380638 & 0.870973 \\
0.9 & 2.639800 & 1.612977 & 0.146483 & 1.213717 & 4.387023 & 0.867323 \\
\hline
\end{tabular}

\begin{tabular}{llllll}
\hline$p$ & $E A$ & RRATE & EUR & EUS & COST \\
\hline 0.1 & 2.405319 & 1.401584 & 4.151391 & 0.363537 & 100.2953 \\
0.2 & 2.405389 & 1.401625 & 4.151512 & 0.359440 & 100.1917 \\
0.3 & 2.405456 & 1.401664 & 4.151628 & 0.355589 & 100.0942 \\
0.4 & 2.405520 & 1.401702 & 4.151738 & 0.351962 & 100.0025 \\
0.5 & 2.405581 & 1.401737 & 4.151844 & 0.348539 & 99.9159 \\
0.6 & 2.405640 & 1.401771 & 4.151945 & 0.345305 & 99.8340 \\
0.7 & 2.405696 & 1.401804 & 4.152041 & 0.342243 & 99.7565 \\
0.8 & 2.405749 & 1.401835 & 4.152133 & 0.339339 & 99.6830 \\
0.9 & 2.405801 & 1.401865 & 4.152223 & 0.336583 & 99.6133 \\
\hline
\end{tabular}

Table 7: Numerical results for $S=4, s=3$. 


\section{Acknowledgements}

The authors thank the referees for their valuable suggestions and comments.

\section{References}

[1] Nahmias S, 1982, Perishable inventory theory, Operations Research, 30(4), pp. 680708.

[2] RaAfat F, 1991, Survey of literature on continuously deteriorating inventory models, Journal of the Operations Research Society, 42, pp. 27-37.

[3] Yadavalli VSS, Natarajan R, Hemamalini L \& Hargreaves CA, 2001, Stochastic model of a two-product inventory system with product interaction, Management Dynamics, 10(3), pp. 81-91.

[4] YADAVAlLi VSS \& JOUBERT JW, 2003, A two-product single period manufacturing and supply system, Management Dynamics, 12(2), pp. 34-39.

[5] Yadavalli VSS, Anbazhagan N \& Arivarignan G, 2004, A two-commodity stochastic inventory system with lost sales, Stochastic Analysis and Applications, 22(2), pp. 479-494. 
